$\begin{array}{cc}\text { ACADEMIA ROMÂNĂ } & \text { Rev. Roum. Chim., } \\ \text { Revue Roumaine de Chimie } & \text { 2019, 64(7), 585-593 } \\ \text { http://web.icf.ro/rrch/ } & \text { DOI: 10.33224/rrch.2019.64.7.05 }\end{array}$

\title{
BIGINELLI REACTION VIA BIS-UREIDE INTERMEDIATE IN LOW MELTING MIXTURE
}

\author{
Hitesh MAHAJAN, Babita JAMWAL and Satya PAUL * \\ Department of Chemistry, University of Jammu, Jammu-180 006, India
}

Received November 17, 2017

This paper deals with the study of mechanism of the Biginelli reaction using low melting mixture as solvent and catalyst. The catalytic effect of low melting mixture and the reaction mechanism is discussed on the basis of nuclear magnetic resonance (NMR) and mass spectrometry (ESIMS). All experimental measurements point to a mechanism involving bis-ureide derivative as a key intermediate, formed by the reaction of benzaldehyde and urea in low melting mixture.

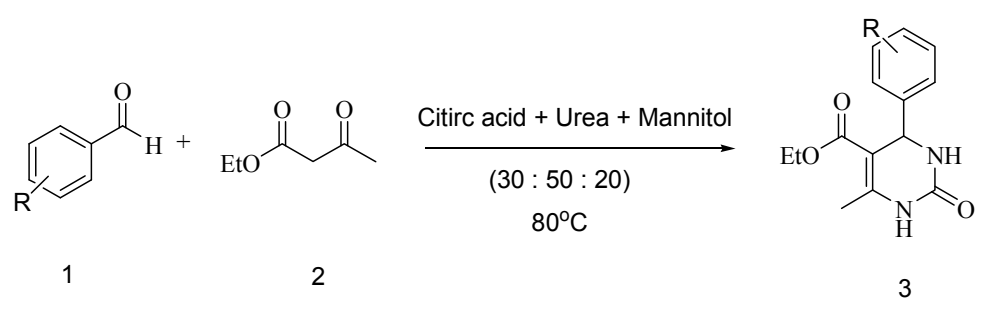

\section{INTRODUCTION}

Multicomponent reactions (MCRs) are reactions of three or more reactants in one-pot, leading to a product that ideally contains all atoms of the reactants. ${ }^{1-5}$ These reactions have great impact in organic synthesis especially in the formation of carbon-carbon and carbon-heteroatom bonds in one-pot involving simple reaction procedures, high atom economy and high selectivity in generating molecular complexity in a single synthetic operation. Owing to their bond forming efficiency, MCRs are the preferred approach in the drug discovery processes.

Especially in the last two decades, the chemistry and biology of 3,4-dihydropyrimidin$2(1 H)$-ones (or thiones) DHPMs, have return to prominence. Large number of important reviews dealing with the synthesis of DHPMs using new catalysts and solvents, along with the study of their biological and medicinal effects have been carried out. ${ }^{6-16}$ DHPM derivatives (Fig. 1) have large biological activities and many synthetic samples have been studied as calcium channel modulators, mitotic kinesin inhibitors, adrenergic receptor antagonists, antibacterials, antivirals, and others. $^{17,18}$ Some natural products containing DHPM moiety have been studied as new leads for AIDS therapies.

The Biginelli multicomponent reaction (MCR), first reported by Pietro Biginelli (Scheme 1) in $1891^{19}$ is the most elegant and direct methodology to obtain 3,4-dihydropyrimidin-2(1H)-one (DHPMs) derivatives in single-step procedure. This three component one-pot reaction leads to the synthesis of dihydropyrimidines, typically by the reaction of a benzaldehyde, an acetoacetate and (thio)urea under acid catalysis. ${ }^{20}$ 
<smiles>CCCCOC(=O)C1=C(C)NC(=O)NC1c1cccc(O)c1</smiles>

Monastrol<smiles>CCOC(=O)C1=C(C)NC(=O)NC1c1ccc2c(c1)OCO2</smiles>

Piperastrol<smiles>O=C1NC2=C(C(=O)CCC2)C(c2cccc(O)c2)N1</smiles>

Enastron

Fig. 1 - Some biologically active DHPMs.

Bignelli reactions can be performed under a variety of conditions, and several advancements of the experimental procedures have been reported in recent years. Although it has been traditionally catalyzed by strong Bronsted acids, Lewis acids ${ }^{21-25}$ are now being increasingly used. Besides these, DHPMs have also been synthesised using ionic liquids or supported ionic liquids, ${ }^{26}$ and so on. They have also been synthesized under microwave conditions $^{27}$ and ultrasound irradiations. ${ }^{28}$ Indeed, what is nowadays known as eponymous reaction was born as a controversial reaction, since the original proposed structure for the Biginelli adduct was wrongly assigned and had to be later revisited. ${ }^{29}$

\section{General Scope}

Somehow, controversies surrounding the Biginelli reaction are still hotly debated. For instance, there is no consensus about the actual mechanism for this transformation, and at least three propositions are well accepted and discussed.
Another controversial issue related to the Biginelli reaction is the solvent effect. Despite being considered to be an effective reaction due to its multicomponent character, many of the developed conditions require the use of toxic and expensive solvents, therefore contrasting with the green and promising features of the Biginelli reaction. In this sense, alternative solvents or media such as ionic liquids (ILs), PEG and water have been successfully tested.

Recently, low melting mixtures of carbohydrates, urea or $N, N$-dimethylurea and inorganic salts (optional) have been reported as alternative solvents for various organic transformations. ${ }^{30}$ These stable melts are environmentally benign, being easily biodegradable, relatively non-toxic and available from bulk renewable resources without numerous energy consuming modification steps. They have low vapor pressure and good solvent properties and have been successfully used in the Diels-Alder reaction, cycloadditions, Stille and Suzuki cross-couplings, hydrogenation reactions ${ }^{31}$ and in the synthesis of monosubstituted ureas. ${ }^{32}$

General Scope

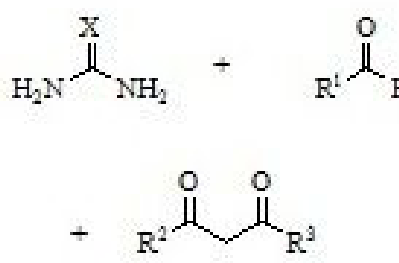

$\mathrm{R}^{1}$ or $\mathrm{R}^{2}$ or $\mathrm{R}^{3}=\mathrm{alky1}$ or aryl $; \mathrm{X}=0, \mathrm{~S}$

First reaction performed by Biginelli in 1891

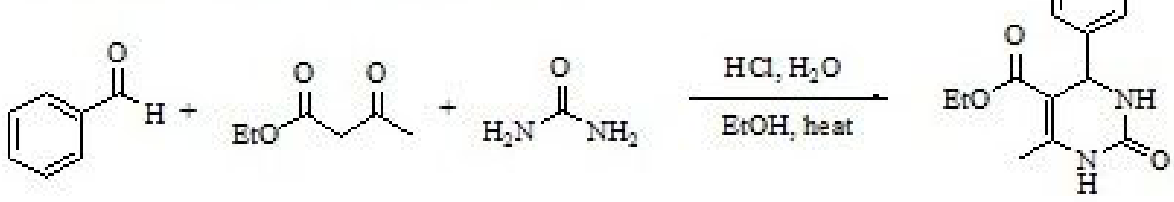

Scheme 1 - General scope of the Biginelli reaction. 


\section{RESULTS AND DISCUSSION}

Due to our interest in studying the mechanism of Biginelli reaction in low melting mixture, we initially tested the standard Biginelli reaction by mixing benzaldehyde ( $1 \mathrm{mmol}, 1)$, ethyl acetoacetate (1 mmol, 2) in low melting citric acid, urea and mannitol melt at $80{ }^{\circ} \mathrm{C}$, to get the desired product 3 (Scheme 2).

It is important to note that the low melting mixture played an important role of solvent as well as catalyst in Biginelli reaction. To select the best organic melt in terms of reaction time, selectivity and yield, various organic melts have been prepared and among them, melt of citric acid, urea and mannitol gave the best results at $80^{\circ} \mathrm{C}$ (Table 1$)$.
In order to investigate the mechanism of the Biginelli reaction, we make use of electrospray ionization mass spectroscopy, as it is capable of "fishing" the formed ions from the solution, leading them directly to the gas phase. Fortunately, we were able to detect and characterize interesting intermediates and transient species, as will be shown and discussed.

We first investigated the formation and isolation of dormant bisureide derivative. The first step of this route is the reaction between protonated urea coming from the melt and 4methylbenzaldehyde at $80^{\circ} \mathrm{C}$ in the absence of ethyl acetoacetate (Scheme 3). Two different pathways involving $\mathrm{C}-\mathrm{O}$ and $\mathrm{C}-\mathrm{N}$ bond formation could have been possible (Fig. 2).<smiles>[R]c1ccc(C([CH-])=O)cc1</smiles>

1<smiles>CCOC(=O)CC(C)=O</smiles>

2

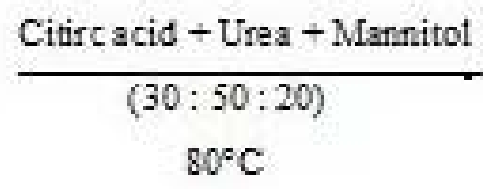

$80^{\circ} \mathrm{C}$<smiles>[R][X]c1cccc(C2NC(=O)NC(C)=C2C(=O)OCC)c1</smiles>

3

Scheme 2 - Biginelli reaction in low melting mixture.

Table 1

Efficacy of different organic melts in the synthesis of 3,4-dihydropyrimidin-2(1H)-ones ${ }^{\mathrm{a}}$

\begin{tabular}{|c|c|c|c|c|}
\hline S.No & Organic Melt & M.Pt $\left({ }^{\circ} \mathrm{C}\right)$ & Time (h) & Yield $^{\mathbf{b}}(\%)$ \\
\hline 1 & Tartaric acid+Mannitol+Urea $(30: 20: 50)$ & 65 & 15 & 87 \\
\hline 2 & Glucose+Urea $+\mathrm{NH}_{4} \mathrm{Cl}(60: 30: 10)$ & 79 & 22 & 60 \\
\hline 3 & Tartaric acid+Fructose+Urea $(30: 20: 50)$ & 75 & 17 & 83 \\
\hline 4 & Fructose+Urea (50:50) & 65 & 20 & 74 \\
\hline 5 & Citric acid+Mannitol+Urea (30:20:50) & 80 & 12 & 92 \\
\hline
\end{tabular}

${ }^{\mathrm{a}}$ Reaction conditions: benzaldehyde $(1 \mathrm{mmol})$, ethyl acetoacetate $(1 \mathrm{mmol})$ and organic melt $(2.5 \mathrm{~g})$.

${ }^{\mathrm{b}}$ Isolated yields refer to the yields obtained by the crystallization from ethyl acetate:petroleum ether.

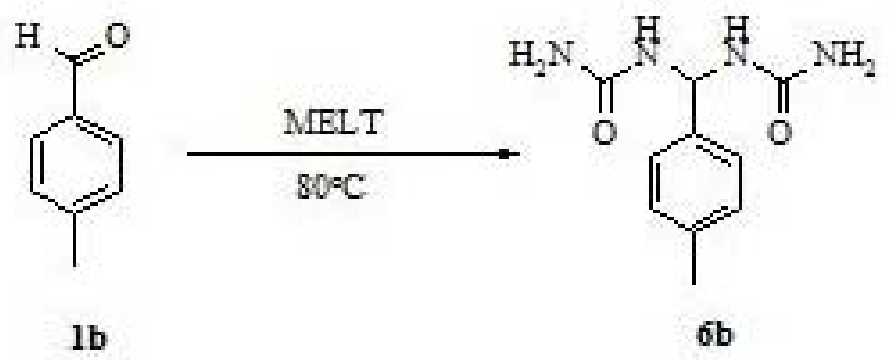

Scheme 3 - Formation of 1,1'-(4-methylphenylmethylene)bisurea derivative. 


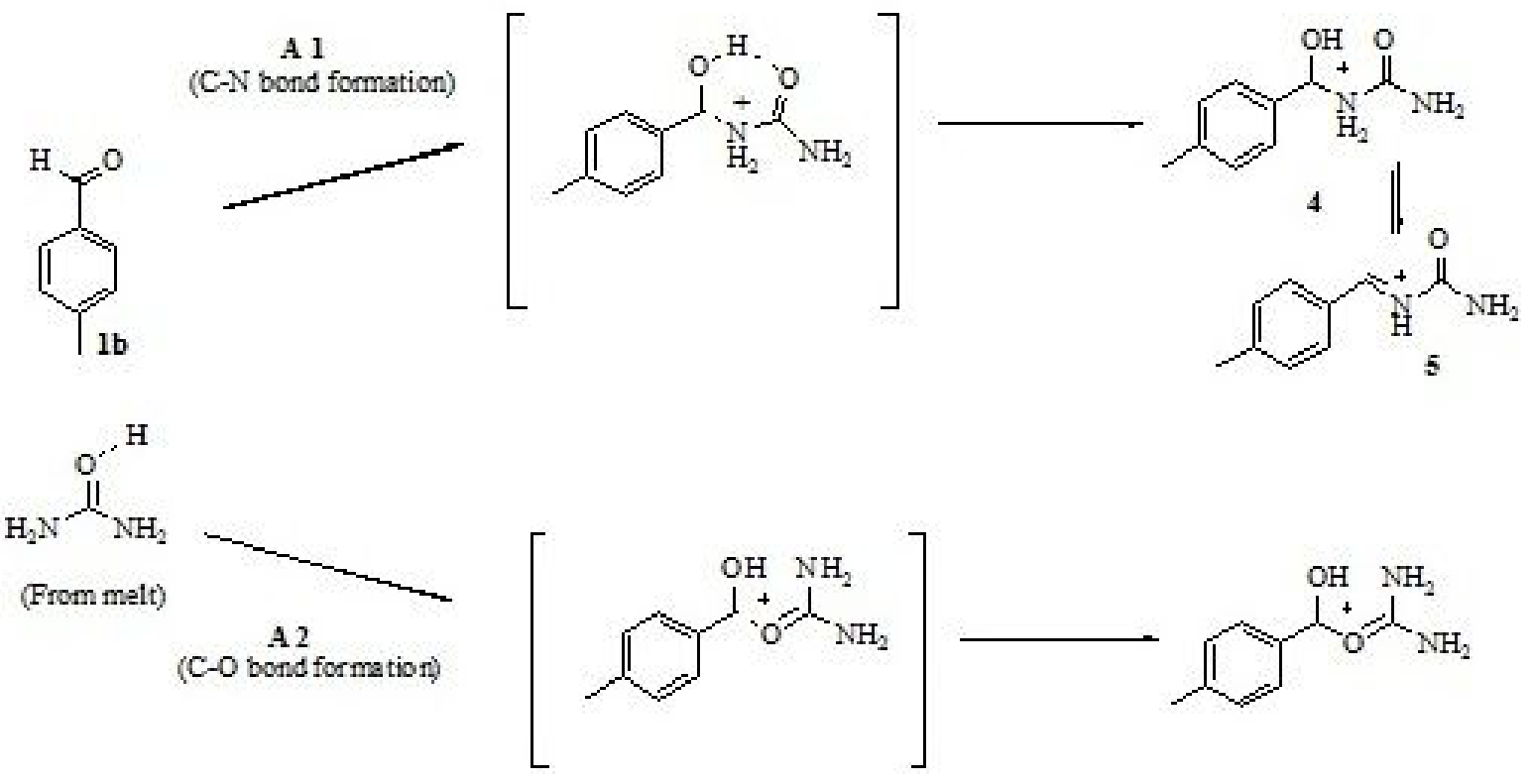

Fig. 2 - Intermediates and transition states between protonated urea and 4-methylbenzaldehyde.

Between them, the lowest energy pathway is the $\mathrm{C}-\mathrm{N}$ bond formation (A1) leading to the formation of an iminium intermediate 5 . In this path, the proton transfer from the urea oxygen to the benzaldehyde oxygen takes place with the $\mathrm{C}-\mathrm{N}$ bond formation between urea amine group and benzaldehyde carbonyl carbon. Formation of bisureide derivative (6b) takes place by the reaction of another urea molecule with 5 . The formation of 4 , 5 and $6 \mathrm{~b}$ was further confirmed by mass spectroscopy (Fig. 3).

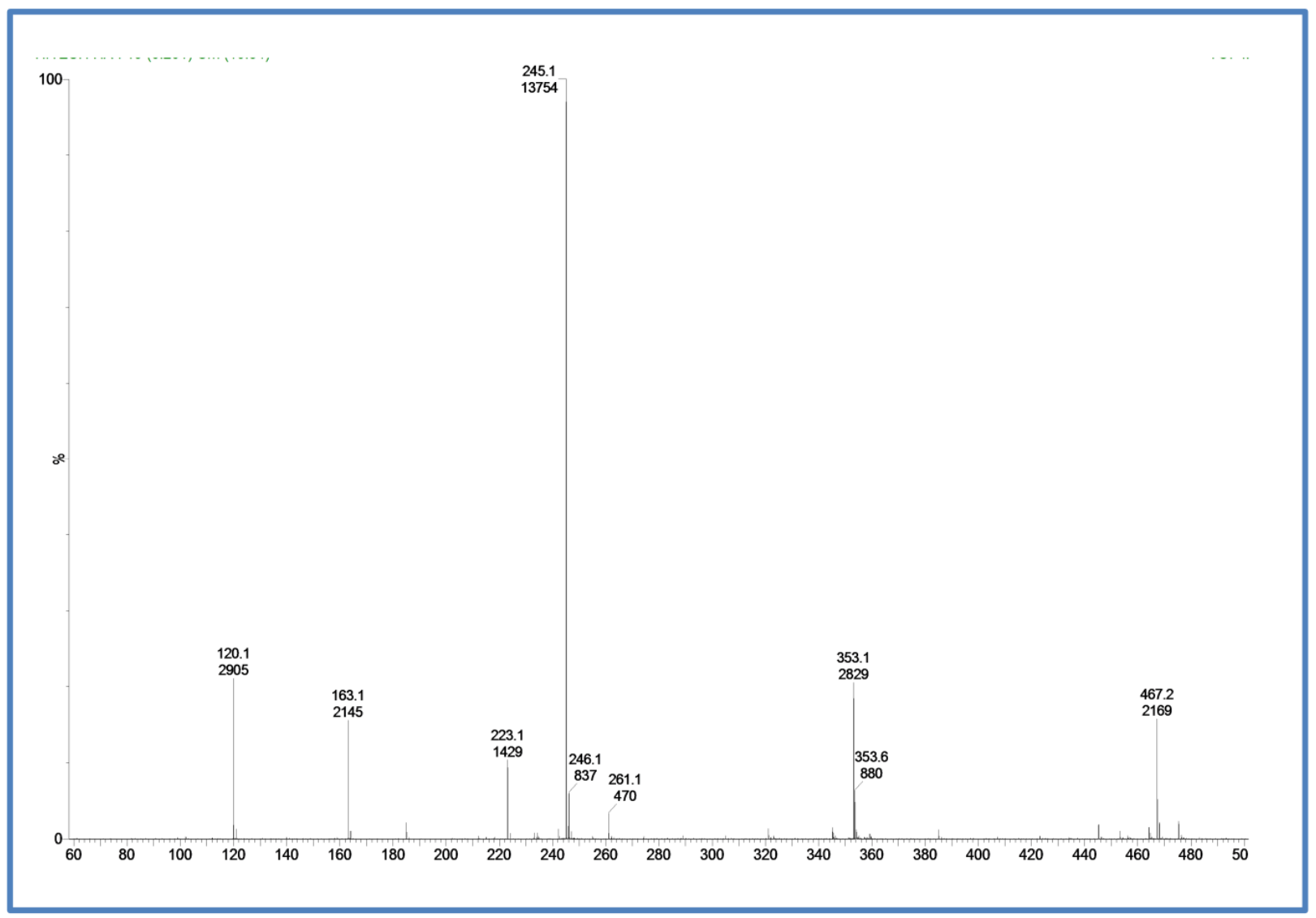

Fig. 3 - ESI (+)-MS of 1,1'-(4-methylphenylmethylene)bisurea derivative. 


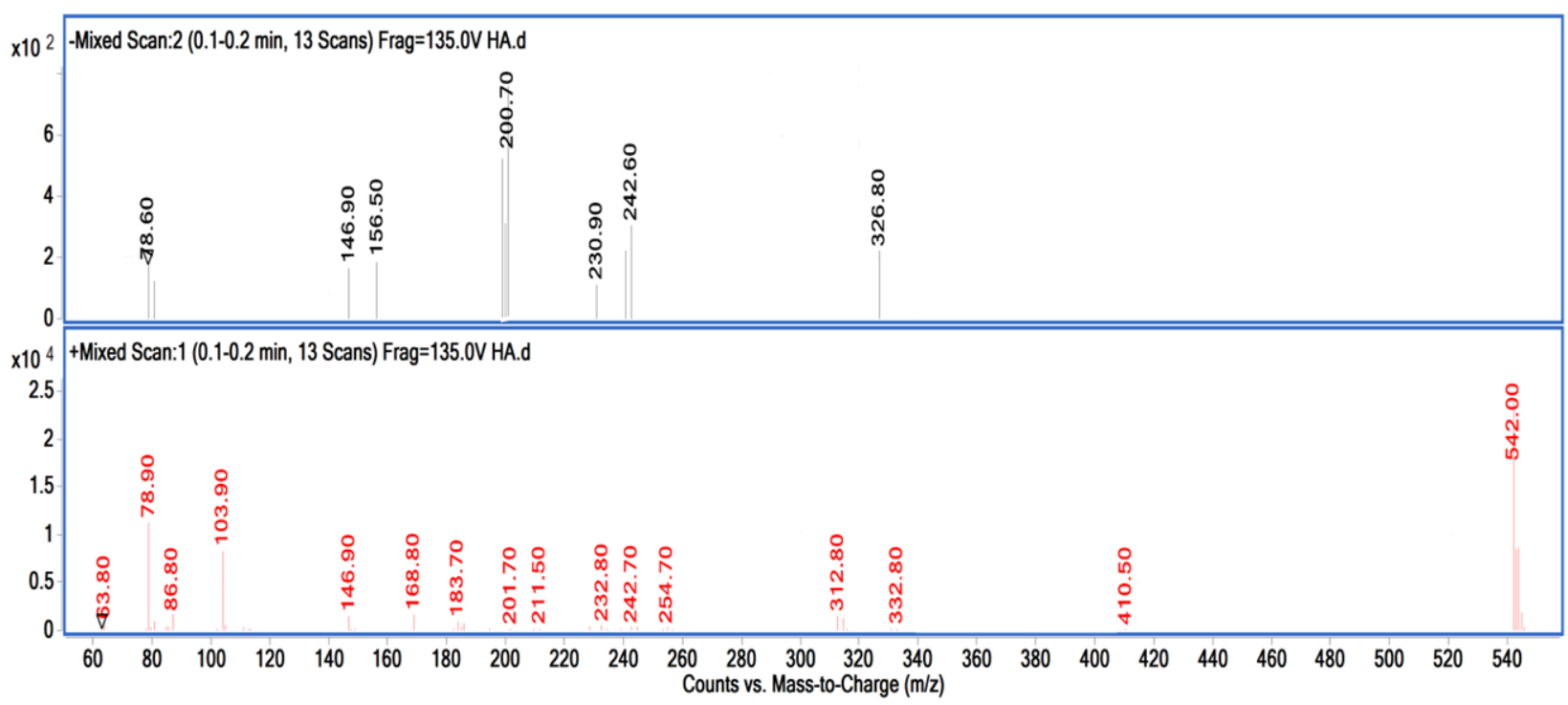

Fig. 4 - ESI (+)-MS of 1,1'-(4-chlorophenylmethylene)bisurea derivative.

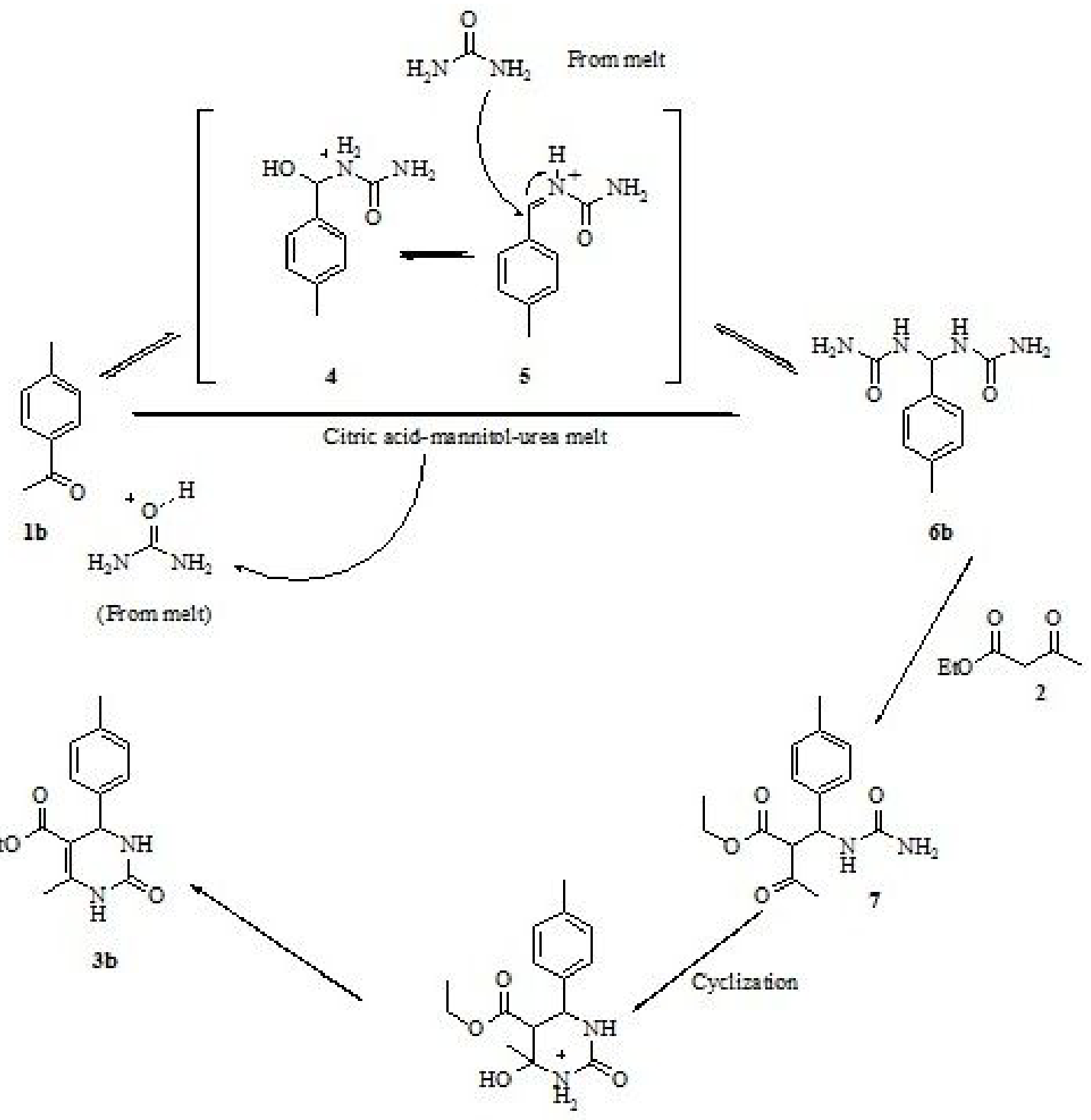

Fig. 5 - Proposed mechanism of Biginelli reaction using low melting mixture. 
Figure 3 shows that ESI(+)-MS was able to intercept key cationic species: protonated bisureide $\left[6 b+\mathrm{H}^{+}\right]$of $\mathrm{m} / \mathrm{z} 223$; the iminium ion [5] of $\mathrm{m} / \mathrm{z}$ 163; its hydrated precursor [4] of $\mathrm{m} / \mathrm{z} 181$, protonated 4-methylbenzaldehyde $\left[1 \mathrm{~b}+\mathrm{H}^{+}\right]$of $\mathrm{m} / \mathrm{z}$ 121. Similar peaks were observed in the ESI(+)MS of 1,1'-(4-chlorophenylmethylene)bisurea derivative (Fig. 4).

After the formation of bis-ureide derivative $(\mathbf{6 b})$, the C-C bond formation between $\mathbf{6 b}$ and ethyl acetoacetate was considered. In solution, $\beta$ keto ester can tautomerize into the corresponding enol form. Although the keto form is more stable, the enol form is needed for $\mathrm{C}-\mathrm{C}$ bond formation with $\mathbf{6 b}$ leading to the formation of 7 . The transformation of $\mathbf{7}$ to final product $\mathbf{3 b}$ takes place via cyclization, followed by dehydration and deprotonation step. The most plausible reaction mechanism for the formation of 3,4dihydropyrimidin-2(1H)-ones using low melting mixture is shown in Fig. 5.
It is further noted that the presence of extra urea in the melt does not directly participate in the mechanism, however it may stabilize the resulting product as it may work as a strong proton acceptor from the ethyl acetoacetate. Using this hypothesis, we have isolated number of bis-uriede derivatives by the reaction of various aldehydes having electron-releasing and electron-withdrawing groups in melt and used them for the preparation of various 3,4-dihydropyrimidin-2 $(1 \mathrm{H})$-ones (Table $2)$. All the reactions proceeded efficiently and afforded products in excellent yields. This very simple and convenient experimental procedure tolerated a variety of electron-releasing and electron-withdrawing groups under the present reaction conditions. The products obtained were confirmed by comparing their melting point with authentic samples and by ${ }^{1} \mathrm{H}$ and ${ }^{13} \mathrm{C}$ NMR spectral data. The importance of the present work lies in the fact that it supports the iminium mechanism, which proceeds via bis-ureide as a key intermediate in Bignelli reaction.

Table 2

Synthesis of various 3,4-dihydropyrimidin-2(1H)-ones ${ }^{\mathrm{a}}$ using citric acid-mannitol-urea melt $\mathrm{t}^{\mathrm{a}}$

\begin{tabular}{llllll}
\hline S.No & $\mathbf{R}$ & Product & Bis-ureide & Time (h) & Yield $^{\mathbf{b}} \mathbf{( \% )}$ \\
\hline 1 & $-\mathrm{H}$ & $\mathbf{3 a}$ & $\mathbf{6 a}$ & 12 & 92 \\
2 & $4-\mathrm{CH}_{3}$ & $\mathbf{3 b}$ & $\mathbf{6 b}$ & 10 & 90 \\
3 & $4-\mathrm{OCH}_{3}$ & $\mathbf{3 c}$ & $\mathbf{6 c}$ & 14 & 89 \\
4 & $4-\mathrm{Cl}$ & $\mathbf{3 d}$ & $\mathbf{6 d}$ & 11 & 91 \\
5 & $2-\mathrm{Cl}$ & $\mathbf{3 e}$ & $\mathbf{6 e}$ & 13 & 90 \\
6 & $2-\mathrm{NO}_{2}$ & $\mathbf{3 f}$ & $\mathbf{6 f}$ & 13 & 87 \\
7 & $3-\mathrm{NO}_{2}$ & $\mathbf{3 g}$ & $\mathbf{6 g}$ & 12 & 85 \\
8 & $4-\mathrm{Br}^{2}$ & $\mathbf{3 h}$ & $\mathbf{6 h}$ & 14 & 87 \\
9 & $4-\mathrm{NO}_{2}$ & $\mathbf{3 i}$ & $\mathbf{6 i}$ & 9 & 91 \\
10 & $4-\mathrm{OH}$ & $\mathbf{3 j}$ & $\mathbf{6 j}$ & 18 & 79 \\
11 & Furfyl & $\mathbf{3 k}$ & $\mathbf{6 k}$ & 19 & 76 \\
12 & $\mathrm{Thienyl}$ & $\mathbf{3 l}$ & $\mathbf{6 l}$ & 18 & 75 \\
13 & $2,4-\mathrm{Cl}_{2}$ & $\mathbf{3 m}$ & $\mathbf{6 m}$ & 15 & 79 \\
\hline
\end{tabular}

${ }^{\mathrm{a}}$ Reaction conditions: arylaldehyde $(1 \mathrm{mmol})$, ethyl acetoacetate $(1 \mathrm{mmol})$, melt $(2 \mathrm{~g})$ at $80{ }^{\circ} \mathrm{C}$.

${ }^{\mathrm{b}}$ Isolated yields refer to the yields obtained by the crystallization from ethyl acetate:petroleum ether. 


\section{EXPERIMENTAL}

\section{Materials and Methods}

All chemicals were purchased as reagent grade and used without further purification, unless otherwise mentioned. The structures of the products were confirmed by IR, ${ }^{1} \mathrm{H}$ NMR, mass spectral data and by comparison with authentic samples available commercially or prepared according to the literature methods. Nuclear magnetic resonance $\left({ }^{1} \mathrm{H}\right.$ NMR and ${ }^{13} \mathrm{C}$ NMR) spectra were recorded in DMSO- $d_{6}$ on Bruker DPX (400 MHz) spectrometer using TMS as an internal standard. The mass spectra were obtained on Esquire 3000 Bruker Daltonics spectrometer (ESI). TLCs were carried out on silica gel plates which were activated at $100^{\circ} \mathrm{C}$ for $1 \mathrm{~h}$ and stored in a dessicator. Iodine vapors were used for the visualization of spots.

\section{General procedure for the preparation of DHPMs via bis-ureides}

The constituents of the melt (citric acid-0.75 g, urea-1.25 g and mannitol- $0.5 \mathrm{~g}$ ) were ground using a mortar and pestle, and then transferred to a round bottom flask equipped with a stirring bar and condenser, and heated to $80^{\circ} \mathrm{C}$ to form a clear melt. To this melt, 1 mmole of aldehyde was added and stirred for the appropriate time to get a solid product, which was isolated as bis-ureide derivative. Further, 1 mmole of ethyl acetoacetate was added to 1 mmole of isolated bis-ureide and stirred for the appropriate time (Table 2). After completion of the reaction [monitored by TLC (EtOAc: pet ether 1:9)], the reaction mixture was cooled to room temperature and diluted with water $(20 \mathrm{ml})$. The product was extracted with EtOAc $(20 \mathrm{~mL})$ and dried over anhydrous $\mathrm{Na}_{2} \mathrm{SO}_{4}$. Removal of the solvent under reduced pressure gave solid product which was purified either by crystallization from ethyl acetate: pet ether or using column chromatography [EtOAc:pet.ether- 0.5:9.5, $\mathrm{SiO}_{2}$ (E. Merck India; 60-200 mesh)].

\section{General procedure for the preparation of DHPMs without isolating bis-ureides}

The constituents of the melt (citric acid- $0.75 \mathrm{~g}$, urea- $1.25 \mathrm{~g}$ and mannitol- $0.5 \mathrm{~g}$ ) were ground using a mortar and pestle and then transferred to a round bottom flask equipped with a stirring bar and condenser, heated to $80^{\circ} \mathrm{C}$ to form a clear melt. To this melt, 1 mmole of aldehyde and $1 \mathrm{mmol}$ of ethyl acetoacetate were added at $80^{\circ} \mathrm{C}$. After completion of the reaction [monitored by TLC (EtOAc: pet ether 1:9)], the reaction mixture was cooled to room temperature and diluted with water $(20 \mathrm{~mL})$. The product was extracted with EtOAc $(20 \mathrm{ml})$ and dried over anhydrous $\mathrm{Na}_{2} \mathrm{SO}_{4}$. Removal of the solvent under reduced pressure gave solid product which was purified either by crystallization from ethyl acetate: pet ether or using column chromatography [EtOAc:pet.ether- 0.5:9.5, $\mathrm{SiO}_{2}$ (E. Merck India; 60-200 mesh)].

\section{Spectral data of 3,4-dihydropyrimidin-2-(1H)-ones ( 3a-m )}

5-(Ethoxycarbonyl)-6-methyl-4-phenyl-3, 4dihydropyrimidin-2-(1H)-one (3a)

M.p ( $\left.{ }^{\circ} \mathbf{C}\right):$ 205-206/206-208. ${ }^{33}$

IR (KBr, $v_{\max }$ in $\left.\mathbf{~ c m}^{-1}\right)$ : 3243, 1726, 1649.

${ }^{1}$ H-NMR (DMSO-d d $_{\mathbf{6}}$ : $\delta 9.19$ (bs, 1H, -NH), 7.74 (bs, 1H, -NH), 7.34-7.23 (m, 5H, Ar-H), 5.15-5.14 (d, 1H, -CH), 4.01$3.95\left(\mathrm{q}, 2 \mathrm{H}-\mathrm{OCH}_{2} \mathrm{CH}_{3}\right), 2.24\left(\mathrm{~s}, 3 \mathrm{H},-\mathrm{CH}_{3}\right), 1.07-1.11(\mathrm{t}, 3 \mathrm{H}$, $-\mathrm{OCH}_{2} \mathrm{CH}_{3}$ ).
${ }^{13}$ C-NMR (DMSO-d ${ }_{6}$ ): $\delta 165.82,152.62,148.79,145.28$, $128.86,127.75,126.70,99.77,59.68,54.42,18.22,14.52$.

MS (ESI): $261(\mathrm{M}+1)$.

5-(Ethoxycarbonyl)-6-methyl-4-(4-methylphenyl)-3, 4-dihydropyrimidin-2-(1H)-one (3b)

M.p ( $\left.{ }^{\circ} \mathbf{C}\right): 214-215 / 215-216 .^{33}$

IR (KBr, $v_{\max }$ in $\left.\mathbf{c m}^{-1}\right): 3158,1715,1632$.

${ }^{1}$ H-NMR (DMSO-d ${ }_{6}$ ): $\delta 9.18$ (bs, $\left.1 \mathrm{H},-\mathrm{NH}\right), 7.82$ (bs, $1 \mathrm{H}$, $-\mathrm{NH}), 5.11-5.09(\mathrm{~d}, 1 \mathrm{H},-\mathrm{CH}), 4.00-3.96\left(\mathrm{q}, 2 \mathrm{H},-\mathrm{OCH}_{2} \mathrm{CH}_{3}\right)$, $2.76\left(\mathrm{~s}, 3 \mathrm{H},-\mathrm{CH}_{3}\right), 2.18\left(\mathrm{~s}, 3 \mathrm{H},-\mathrm{CH}_{3}\right), 1.11-1.08(\mathrm{t}, 3 \mathrm{H}$, $\left.-\mathrm{OCH}_{2} \mathrm{CH}_{3}\right)$.

${ }^{13}$ C-NMR (DMSO-d ${ }_{6}$ ): $\delta$ 165.56, 156.42, 151.92, 148.39, $134.78,125.65,123.26,101.02,60.61,53.65,34.26,18.26$, 14.51 .

MS (ESI): $275(\mathrm{M}+1)$.

5-(Ethoxycarbonyl)-4-(4-methoxyphenyl)-6-methyl-3, 4dihydropyrimidin-2-(1H)-one (3c)

M.p ( ${ }^{\circ}$ C): 202-203/201-202. ${ }^{34}$

IR (KBr, $v_{\max }$ in $\left.\mathbf{c m}^{-1}\right)$ : 3243, 1725, 1651.

${ }^{1}$ H-NMR (DMSO-d ${ }_{6}$ ): $\delta 9.15$ (bs, $\left.1 \mathrm{H},-\mathrm{NH}\right), 7.67$ (bs, $1 \mathrm{H}$, -NH), 7.15-7.13 (d, 2H, Ar-H), 6.88-6.86 (d, 2H, Ar-H), 5.095.08 (d, $1 \mathrm{H},-\mathrm{CH}), 3.98-3.96$ (q, $\left.2 \mathrm{H},-\mathrm{OCH}_{2} \mathrm{CH}_{3}\right), 3.37$ (s, 3H, $\left.-\mathrm{OCH}_{3}\right), 2.24\left(\mathrm{~s}, 3 \mathrm{H},-\mathrm{CH}_{3}\right), 1.12-1.08\left(\mathrm{t}, 3 \mathrm{H},-\mathrm{OCH}_{2} \mathrm{CH}_{3}\right)$.

${ }^{13}$ C-NMR (DMSO-d ${ }_{6}$ ): $\delta 165.84,158.91,152.61,148.46$, $137.51,127.85,114.17,100.04,59.61,55.52,53.79,18.21$, 14.57 .

MS (ESI): $291(\mathrm{M}+1)$.

5-(Ethoxycarbonyl)-4-(4-chlorophenyl)-6-methyl-3, 4-dihydropyrimidin-2-(1H)-one (3d)

M.p ( $\left.{ }^{\circ} \mathbf{C}\right):$ 214-215/215-216. ${ }^{34}$

IR (KBr, $v_{\max }$ in $\left.\mathbf{c m}^{-1}\right)$ : 3225, 1720, 1615.

${ }^{1}$ H-NMR (DMSO-d $\left.)_{6}\right): \delta: 9.05(\mathrm{~s}, 1 \mathrm{H}, \mathrm{NH}), 8.19-8.16(\mathrm{~d}, 2 \mathrm{H}$, Ar-H), 7.69 (s, 1H, NH), 7.55-7.51 (d, 2H, Ar-H), 5.65-5.60 (d, $1 \mathrm{H}-\mathrm{CH}), 4.03-3.96$ (q, $\left.2 \mathrm{H}, \mathrm{OCH}_{2} \mathrm{CH}_{3}\right), 2.32\left(\mathrm{~s}, 3 \mathrm{H}, \mathrm{CH}_{3}\right)$.

${ }^{13}$ C-NMR (DMSO-d 6 ): $\delta: 165.85,159.15,153.41,152.26$, 138.34, 130.37, 118.15, 101.60, 60.15, 55.81, 18.71, 14.22 . MS (ESI): $295(\mathrm{M}+1)$.

5-(Ethoxycarbonyl)-4-(2-chlorophenyl)-6-methyl-3, 4-dihydropyrimidin-2-(1H)-one $(3 \mathrm{e})$

M.p ( $\left.{ }^{\circ} \mathbf{C}\right):$ 216-217/216-218. ${ }^{34}$

IR (KBr, $v_{\max }$ in $\left.\mathbf{c m}^{-1}\right): 3232,1725,1620$.

${ }^{1}$ H-NMR (DMSO-d $\left.{ }_{6}\right): \quad \delta: 9.30(\mathrm{~s}, 1 \mathrm{H}, \mathrm{NH}), 8.00(\mathrm{~s}, 1 \mathrm{H}$, $\mathrm{NH}), 7.28-7.16(\mathrm{~m}, 4 \mathrm{H}, \mathrm{Ar}-\mathrm{H}), 5.28-5.24(\mathrm{~d}, 1 \mathrm{H}, \mathrm{CH}), 4.04-$ 3.92 (q, $\left.2 \mathrm{H}, \mathrm{OCH}_{2} \mathrm{CH}_{3}\right), 2.16\left(\mathrm{~s}, 3 \mathrm{H}, \mathrm{CH}_{3}\right), 1.09-1.06$ (t, $3 \mathrm{H}$, $\left.\mathrm{OCH}_{2} \mathrm{CH}_{3}\right)$.

${ }^{13}$ C-NMR (DMSO-d 6 ): $\delta: 166.4,155.5,146.2,143.2,129.4$, 126.1, 122.2, 100.2, 60.9, 54.4, 17.4, 14.2.

MS (ESI): $295(\mathrm{M}+1)$.

5-(Ethoxycarbonyl)-4-(2-nitrophenyl)-6-methyl-3, 4-dihydropyrimidin-2-(1H)-one (3f)

M.p ( $\left.{ }^{\circ} \mathbf{C}\right): 208-210 / 208-210 .{ }^{33}$

IR (KBr, $v_{\text {max }}$ in $\left.\mathbf{~ c m}^{-1}\right)$ : 3237, 1730, 1618.

${ }^{1}$ H-NMR (DMSO-d ${ }_{6}$ ): $\delta 9.36$ (bs, $\left.1 \mathrm{H},-\mathrm{NH}\right), 7.90$ (bs, $1 \mathrm{H}$, -NH), 8.20-7.42 (m, 4H, Ar-H), 5.29-5.27 (d, 1H, -CH), 4.00$3.96\left(\mathrm{q}, 2 \mathrm{H},-\mathrm{OCH}_{2} \mathrm{CH}_{3}\right), 2.26\left(\mathrm{~s}, 3 \mathrm{H},-\mathrm{CH}_{3}\right), 1.11-1.07$ (t, $3 \mathrm{H}$, $-\mathrm{OCH}_{2} \mathrm{CH}_{3}$ ).

${ }^{13}$ C-NMR (DMSO-d $\mathbf{6}$ ): $\delta 165.16,152.74,150.17,149.05$, $138.08,133.53,128.13,128.08,124.32,98.66,60.03,54.08$, $18.33,14.52$.

MS (ESI): $306(\mathrm{M}+1)$.

5-(Ethoxycarbonyl)-4-(3-nitrophenyl)-6-methyl-3,4-dihydropyrimidin-2-(1H)-one $(3 \mathrm{~g})$

M.p ( $\left.{ }^{\circ} \mathbf{C}\right):$ 228-229/227-229. ${ }^{33}$

IR (KBr, $v_{\max }$ in $\left.\mathbf{c m}^{-1}\right): 3229,1724,1630$. 
${ }^{1}$ H-NMR (DMSO-d $)$ ): $\delta 9.36$ (bs, $\left.1 \mathrm{H},-\mathrm{NH}\right), 8.13$ (bs, $1 \mathrm{H}$, -NH), 7.90-7.64 (m, 4H, Ar-H), 5.31-5.30 (d, 1H, -CH), 3.98$3.96\left(\mathrm{q}, 2 \mathrm{H},-\mathrm{OCH}_{2} \mathrm{CH}_{3}\right), 2.28\left(\mathrm{~s}, 3 \mathrm{H},-\mathrm{CH}_{3}\right), 1.12-1.08(\mathrm{t}, 3 \mathrm{H}$, $\left.-\mathrm{OCH}_{2} \mathrm{CH}_{3}\right)$.

${ }^{13}$ C-NMR (DMSO-d ${ }_{6}$ ): $\delta 165.54,152.25,149.89,148.20$ 147.44, 133.46, 130.71, 122.84, 121.46, 98.81, 59.88, 54.00, $18.31,14.47$.

MS (ESI): $306(\mathrm{M}+1)$.

5-(Ethoxycarbonyl)-4-styryl-6-methyl-3,4-dihydropyrimidin2-(1H)-one (3h)

M.p $\left({ }^{\mathbf{O} C)}\right.$ : $230-231 / 230-232 .^{33}$

IR (KBr, $v_{\text {max }}$ in $\left.\mathbf{~ c m}^{-1}\right): 3242,1702,1652$.

${ }^{1}$ H-NMR (DMSO-d (D) $^{-} \delta 9.14$ (bs, $\left.1 \mathrm{H},-\mathrm{NH}\right), 7.55$ (bs, $1 \mathrm{H}$, $-\mathrm{NH}), 7.84-7.52(\mathrm{~m}, 5 \mathrm{H}, \mathrm{Ar}-\mathrm{H}), 6.33-6.28(\mathrm{~d}, 1 \mathrm{H}, \mathrm{H}-\mathrm{C}=\mathrm{CH})$, 6.16-6.10 (dd, $1 \mathrm{H},-\mathrm{CH}=\mathrm{C}-\mathrm{H}), 4.75-4.72(\mathrm{~d}, 1 \mathrm{H},-\mathrm{CH}), 4.10$ $4.04\left(\mathrm{q}, 2 \mathrm{H},-\mathrm{OCH}_{2} \mathrm{CH}_{3}\right), 2.28\left(\mathrm{~s}, 3 \mathrm{H},-\mathrm{CH}_{3}\right), 1.02-1.00(\mathrm{t}, 3 \mathrm{H}$, $-\mathrm{OCH}_{2} \mathrm{CH}_{3}$ ).

${ }^{13}$ C-NMR (DMSO-d ${ }_{6}$ ): $\delta 165.69,153.08,149.00,136.68$, $130.40,129.15,128.59,128.06,126.79,98.27,59.71,52.33$, $18.21,14.71$.

MS (ESI): $287(\mathrm{M}+1)$

5-(Ethoxycarbonyl)-4-(4-nitrophenyl)-6-methyl-3, 4-dihydropyrimidin- 2-(1H)-one (3i)

M.p ( $\left.{ }^{\mathbf{C}} \mathbf{C}\right): 208-210 / 211-213$. $^{33}$

IR (KBr, $v_{\max }$ in $\left.\mathbf{c m}^{-1}\right): 3235,1740,1631$.

${ }^{1} \mathbf{H}-N M R$ (DMSO-d $)$ ): $\delta 9.37$ (bs, $\left.1 \mathrm{H},-\mathrm{NH}\right), 8.23-8.21$ (d, 2H, Ar-H), 7.90 (bs, 1H, -NH), 7.52-7.49 (d, 2H, Ar-H), 5.29$5.27(\mathrm{~d}, 1 \mathrm{H},-\mathrm{CH}), 3.99-3.97\left(\mathrm{q}, 2 \mathrm{H},-\mathrm{OCH}_{2} \mathrm{CH}_{3}\right), 2.08(\mathrm{~s}, 3 \mathrm{H}$, $\left.-\mathrm{CH}_{3}\right), 1.11-1.07\left(\mathrm{t}, 3 \mathrm{H},-\mathrm{OCH}_{2} \mathrm{CH}_{3}\right)$.

${ }^{13}$ C-NMR (DMSO-d ${ }_{6}$ ): $\delta 165.54,152.23,149.86,147.16$, 128.13, 124.31, 98.64, 59.91, 54.11, 31.15, 18.32, 14.49.

MS (ESI): $306(\mathrm{M}+1)$.

5-(Ethoxycarbonyl)-4-(4-hydroxy-3-methoxyphenyl)-6methyl-3, 4-dihydropyrimidin-2-(1H)-one (3j)

M.p ( $\left.{ }^{\circ} \mathbf{C}\right):$ 226-227/227-228. ${ }^{33}$

IR (KBr, $v_{\max }$ in $\left.\mathbf{c m}^{-1}\right): 3374,3239,1721,1655$.

${ }^{1} \mathbf{H}-N M R$ (DMSO-d (D) $_{6} \delta 9.31$ (bs, $\left.1 \mathrm{H},-\mathrm{NH}\right), 7.94$ (bs, 1H, $\mathrm{NH}), 7.80-7.62(\mathrm{~m}, 3 \mathrm{H}, \mathrm{Ar}-\mathrm{H}), 5.68(\mathrm{~s}, 1 \mathrm{H},-\mathrm{OH}), 5.42-5.41$ $(\mathrm{d}, 1 \mathrm{H},-\mathrm{CH}), 3.87-3.83\left(\mathrm{q}, 2 \mathrm{H},-\mathrm{OCH}_{2} \mathrm{CH}_{3}\right), 3.42(\mathrm{~s}, 3 \mathrm{H},-$ $\left.\mathrm{OCH}_{3}\right), 2.12\left(\mathrm{~s}, 3 \mathrm{H},-\mathrm{CH}_{3}\right), 1.11-1.09\left(\mathrm{t}, 3 \mathrm{H},-\mathrm{OCH}_{2} \mathrm{CH}_{3}\right)$

${ }^{13}$ C-NMR (DMSO-d $)_{6}$ ): $\delta 167.42,158.69,151.55,146.24$, $142.67,139.18,121.24,117.72,112.32,101.02,59.64,56.41$, $50.18,18.21,14.54$.

MS (ESI): $307(\mathrm{M}+1)$

5-(Ethoxycarbonyl)-4-(2-furfuryl)-6-methyl-3, 4-dihydropyrimidin-2-(1H)-one (3k)

M.p ( ${ }^{\circ}$ C): $200-201 / 203-204 .^{33}$

IR (KBr, $v_{\max }$ in $\left.\mathbf{c m}^{-1}\right): 3225,1695,1640$.

${ }^{1}$ H-NMR (DMSO-d ${ }_{6}$ ): $\delta 9.24$ (bs, $\left.1 \mathrm{H},-\mathrm{NH}\right), 7.75$ (bs, $1 \mathrm{H}$, -NH), 7.54-6.10 (m, 3H, Ar-CH), 5.19 (s, 1H, -CH), 4.09-4.06 $\left(\mathrm{q}, 2 \mathrm{H},-\mathrm{OCH}_{2} \mathrm{CH}_{3}\right), 2.26\left(\mathrm{~s}, 3 \mathrm{H},-\mathrm{CH}_{3}\right), 1.15-1.12(\mathrm{t}, 3 \mathrm{H}$, $\left.-\mathrm{OCH}_{2} \mathrm{CH}_{3}\right)$.

${ }^{13}$ C-NMR (DMSO-d ${ }_{6}$ ): $\delta$ 165.62, 155.94, 152.16, 149.23, $131.18,126.04,121.14,100.12,59.76,48.93,18.25,14.18$.

MS (ESI): $251(\mathrm{M}+1)$.

5-(Ethoxycarbonyl)-4-(2-thienyl)-6-methyl-3,4-dihydro-

pyrimidin-2-(1H)-one $(3 \mathrm{I})$

M.p ( ${ }^{\circ}$ C): $205-206 / 206-208 .^{33}$

IR $\left(\mathrm{KBr}, v_{\max }\right.$ in $\left.\mathbf{c m}^{-1}\right): 3215,1734,1668$.

${ }^{1} \mathbf{H}-N M R$ (DMSO-d ${ }_{6}$ ): $\delta 9.31$ (bs, $\left.1 \mathrm{H},-\mathrm{NH}\right), 7.91$ (bs, $1 \mathrm{H}$, -NH), 7.36-6.89 (m, 3H, Ar-CH), $5.41(1 \mathrm{H}, \mathrm{s},-\mathrm{CH}), 4.08-4.05$ (q, $\left.2 \mathrm{H},-\mathrm{OCH}_{2} \mathrm{CH}_{3}\right), 2.22\left(\mathrm{~s}, 3 \mathrm{H},-\mathrm{CH}_{3}\right), 1.18-1.15(\mathrm{t}, 3 \mathrm{H}$, $-\mathrm{OCH}_{2} \mathrm{CH}_{3}$ ).

${ }^{13}$ C-NMR (DMSO-d $\left.{ }_{6}\right): \delta 165.51,152.72,149.21,149.11$, $127.15,125.10,123.99,100.27,59.85,49.79,18.13,14.61$. MS (ESI): $267(\mathrm{M}+1)$.
5-(Ethoxycarbonyl)-4-(2,4-dichlorophenyl)-6-methyl-3,4dihydropyrimidin-2-(1H)-one $(3 \mathrm{~m})$

M.p ( $\left.{ }^{\circ} \mathbf{C}\right): 245-247 / 248-249 .{ }^{33}$

IR (KBr, $v_{\max }$ in $\left.\mathbf{c m}^{-1}\right)$ : 3255, 1731, 1651.

${ }^{1}$ H-NMR (DMSO-d $\left.{ }_{6}\right): \delta 9.16$ (s, 1H, NH), 7.69 (s, 1H, NH), 7.51-7.21 (m, 3H, Ar-H), 5.90-5.86 (d, $1 \mathrm{H},-\mathrm{CH}), 4.08-4.04$ (q, $\left.2 \mathrm{H}, \mathrm{OCH}_{2} \mathrm{CH}_{3}\right), 2.64$ (s, $\left.3 \mathrm{H}, \mathrm{CH} 3\right), 1.18-1.16(\mathrm{t}, 3 \mathrm{H}$, $\mathrm{OCH}_{2} \mathrm{CH}_{3}$ ).

${ }^{13}$ C-NMR (DMSO-d $\mathbf{d}_{\mathbf{6}}$ ): $\delta 165.75,159.32,153.23,143.25$, $142.52,131.40,129.15,128.59,127.82,126.79,101.56$, $60.02,55.75,18.60,14.20$.

MS (ESI): $329(\mathrm{M}+1)$.

Spectral data of Bis-ureide derivatives (6a-m)

1,1'-(Phenylmethylene) diurea (6a)

${ }^{1}$ H-NMR (DMSO-d d $_{6}$ ): $\delta$ 7.96-7.26 (m, 5H, Ar-H), 6.72-6.70 (d, 2H, -NH), 6.14-6.11 (t, 1H, -CH), $5.68\left(\mathrm{bs}, 4 \mathrm{H},-\mathrm{NH}_{2}\right.$ ).

${ }^{13}$ C-NMR (DMSO-d ${ }_{6}$ ): $\delta 157.9,136.01,128.5,126.7,125.9$, 58.6 .

MS (ESI): $209(\mathrm{M}+1)$.

1,1'-(4-Methylmethylene) diurea (6b)

${ }^{1}$ H-NMR (DMSO-d ${ }_{\mathbf{6}}$ ): $\delta$ 7.22-7.20 (d, 2H, Ar-H), 7.16-7.14 (d, 2H, Ar-H), 6.73-6.71 (d, 2H, -NH), 6.12-6.08 (t, 1H, -CH), 5.68 (bs, $\left.4 \mathrm{H},-\mathrm{NH}_{2}\right), 2.30\left(\mathrm{~s}, 3 \mathrm{H},-\mathrm{CH}_{3}\right)$.

${ }^{13}$ C-NMR (DMSO-d $_{6}$ ): $\delta 157.8,136.2,129.4,128.7,126.04$, $59.05,20.9$.

MS (ESI): $223(\mathrm{M}+1)$.

1,1'-[(4-Methoxyphenyl)methylene]diurea (6c)

${ }^{1}$ H-NMR (DMSO-d ${ }_{6}$ ): $\delta$ 7.40-7.39 (d, 2H, Ar-H), 6.88-6.87 (d, 2H, Ar-H), 6.74-6.72 (d, 2H, -NH), 6.11-6.08 (t, 1H, -CH), 5.70 (bs, $\left.4 \mathrm{H},-\mathrm{NH}_{2}\right), 3.83\left(\mathrm{~s}, 3 \mathrm{H},-\mathrm{OCH}_{3}\right)$.

${ }^{13}$ C-NMR (DMSO-d 6 ): $\delta$ 158.6, 136.7, 129.8, 127.9, 114.4, $60.8,48.8$.

MS (ESI): $239(\mathrm{M}+1)$.

1,1'-[(4-Chlorophenyl)methylene]diurea (6d)

${ }^{1}$ H-NMR (DMSO-d $)_{6}$ ): $\delta$ 7.41-7.39 (d, 2H, Ar-H), 7.34-7.32 (d, $2 \mathrm{H}, \mathrm{Ar}-\mathrm{H}), 6.83-6.81(\mathrm{~d}, 2 \mathrm{H},-\mathrm{NH}), 6.13-6.09(\mathrm{t}, 1 \mathrm{H},-\mathrm{CH})$, 5.73 (bs, $\left.4 \mathrm{H},-\mathrm{NH}_{2}\right)$.

${ }^{13}$ C-NMR (DMSO-d $\mathbf{6}$ ): $\delta 157.8,141.8,131.6,128.02,127.94$, 58.6 .

MS (ESI): $243(\mathrm{M}+1)$.

1,1'-I(2-Chlorophenyl)methylene]diurea (6e)

${ }^{1}$ H-NMR (DMSO-d $\left._{6}\right): \delta$ 7.54-7.52 (d, $\left.1 \mathrm{H}, \mathrm{Ar}-\mathrm{H}\right), 7.45-7.42$ (d, 1H, Ar-H), 7.37-7.20 (m, 2H, Ar-H), 6.75-6.73 (d, 2H, $-\mathrm{NH}), 6.36-6.32(\mathrm{t}, 1 \mathrm{H},-\mathrm{CH}), 5.63\left(\mathrm{bs}, 4 \mathrm{H},-\mathrm{NH}_{2}\right)$.

${ }^{13}$ C-NMR (DMSO-d 6 ): $\delta 157.7,140.06,132.34,129.8,129.4$, 128.1, 127.3, 57.7.

MS (ESI): $243(\mathrm{M}+1)$.

1,1'-[(2-Nitrophenyl)methylene]diurea (6f)

${ }^{1}$ H-NMR (DMSO-d $\left.)_{6}\right): \delta$ 7.87-7.85 (d, 1H, Ar-H), 7.73-7.64 (m, 2H, Ar-H), 7.55-7.51 (t, 1H, Ar-H), 6.88-6.86 (d, 2H, -NH), 6.56-6.53 (t, 1H, -CH), 5.70 (bs, $\left.4 \mathrm{H},-\mathrm{NH}_{2}\right)$.

${ }^{13}$ C-NMR (DMSO-d $\mathbf{d}_{6}$ ): $\delta 157.8,148.5,136.8,133.1,129.0$, 128.9, 124.6, 56.6,

MS (ESI): $254(\mathrm{M}+1)$.

1,1 '-[(3-Nitrophenyl)methylene]diurea $(6 \mathrm{~g})$

${ }^{1}$ H-NMR (DMSO-d $\left.\mathbf{d}_{\mathbf{6}}\right): \delta 8.49(\mathrm{~s}, 1 \mathrm{H}, \mathrm{Ar}-\mathrm{H}), 8.09-8.07(\mathrm{~d}$, $1 \mathrm{H}, \mathrm{Ar}-\mathrm{H}), 7.84-7.82(\mathrm{~d}, 1 \mathrm{H}, \mathrm{Ar}-\mathrm{H}), 7.61-7.59(\mathrm{t}, 1 \mathrm{H}, \mathrm{Ar}-\mathrm{H})$, 6.89-6.87 (d, 2H, -NH), 6.57-6.54 (t, 1H, -CH), 5.70 (bs, 4H, $-\mathrm{NH}_{2}$ ).

${ }^{13}$ C-NMR (DMSO-d 6 ): $\delta$ 162.7, 147.7, 145.3, 132.0, 129.4, 121.9, 121.7, 71.5.

MS (ESI): $254(\mathrm{M}+1)$.

1,1'-[(4-Bromophenyl)methylene]diurea (6h)

${ }^{1}$ H-NMR (DMSO-d 6 ): $\delta$ 7.85-7.84 (d, 2H, Ar-H), 7.54-7.52 (d, 2H, Ar-H), 7.27-7.25 (d, 2H, -NH), 6.09-6.04 (t, 1H, $-\mathrm{CH}), 5.72\left(\mathrm{bs}, 4 \mathrm{H},-\mathrm{NH}_{2}\right.$ ). 
${ }^{13}$ C-NMR (DMSO-d ${ }_{6}$ ): $\delta$ 158.1, 142.6, 131.3, 128.7, 120.4, 59.08 .

MS (ESI): $287(\mathrm{M}+1)$.

1,1'-[(4-Nitrophenyl)methylene]diurea (6i)

${ }^{1}$ H-NMR (DMSO-d ${ }_{6}$ ): $\delta 8.16-8.14(\mathrm{~d}, 2 \mathrm{H}, \mathrm{Ar}-\mathrm{H}), 7.65-7.64$ (d, 2H, Ar-H), 6.89-6.87 (d, 2H, -NH), 6.57-6.54 (t, 1H, -CH), 5.74 (bs, $4 \mathrm{H},-\mathrm{NH}_{2}$ ).

${ }^{13}$ C-NMR (DMSO-d ${ }_{6}$ ): $\delta 162.8,150.5,145.9,127.8,126.5$, 72.5 .

MS (ESI): $254(\mathrm{M}+1)$.

1,1'-[(4-Hydroxyphenyl)methylene]diurea $(6 \mathbf{j})$

${ }^{1}$ H-NMR (DMSO-d ( $_{6}$ : $\delta$ 7.39-7.38 (d, 2H, Ar-H), 7.34-7.33 (d, 2H, Ar-H), 6.74-6.72 (d, 2H, -NH), 6.35-6.32 (t, 1H, -CH), 5.74 (bs, $\left.4 \mathrm{H},-\mathrm{NH}_{2}\right), 5.35(\mathrm{~s}, 1 \mathrm{H}, \mathrm{OH})$.

${ }^{13}$ C-NMR (DMSO-d $)_{6}$ ): $\delta 162.7,156.5,137.5,128.3,115.7$, 72.5 .

MS (ESI): $225(\mathrm{M}+1)$.

1,1'-(Furan-2-ylmethylene)diurea (6k)

${ }^{1}$ H-NMR (DMSO-d $)_{6}$ ): $\delta$ 7.66-7.65 (d, $\left.1 \mathrm{H}, \mathrm{Ar}-\mathrm{H}\right), 6.75-6.73$ (d, 2H, -NH), 6.46-6.43 (m, 2H, Ar-H), 6.32-6.29 (t, $1 \mathrm{H}$, $-\mathrm{CH}), 5.76$ (bs, $4 \mathrm{H},-\mathrm{NH}_{2}$ ).

${ }^{13}$ C-NMR (DMSO-d 6 ): $\delta 162.7,152.5,142.1,110.6,106.7$, 73.5.

MS (ESI): $199(\mathrm{M}+1)$.

1,1'-(Thiophen-2-ylmethylene) diurea (61)

${ }^{1}$ H-NMR (DMSO-d $\left.{ }_{6}\right): \delta$ 7.41-7.40 (d, 1H, Ar-H), 6.76-6.74 (d, 2H, -NH), 6.89-6.83 (m, 2H, Ar-H), 6.31-6.28 (t, 1H, $-\mathrm{CH}), 5.76$ (bs, $4 \mathrm{H},-\mathrm{NH}_{2}$ ).

${ }^{13}$ C-NMR (DMSO-d ${ }_{6}$ ): $\delta 162.7,139.6,127.0,126.7,125.5$, 73.5.

MS (ESI): $215(\mathrm{M}+1)$.

1,1'-I(2,4-Dichlorophenyl)methylene/diurea $(6 \mathrm{~m})$

${ }^{1}$ H-NMR (DMSO-d $\left.{ }_{6}\right): \delta 7.68(\mathrm{~s}, 1 \mathrm{H}, \mathrm{Ar}-\mathrm{H}), 7.26-7.25(\mathrm{~d}, 1 \mathrm{H}$, Ar-H), 7.12-7.11 (d, 1H, Ar-H), 6.75-6.73 (d, 2H, -NH), 6.36$6.32(\mathrm{t}, 1 \mathrm{H},-\mathrm{CH}), 5.73$ (bs, $\left.4 \mathrm{H},-\mathrm{NH}_{2}\right)$.

${ }^{13}$ C-NMR (DMSO-d 6 ): $\delta 162.7,140.9,133.7,133.6,130.2$, 129.7, 126.7, 69.4.

MS (ESI): $277(\mathrm{M}+1)$

\section{CONCLUSIONS}

ESI (+)-MS monitoring of the Biginelli reaction under three- and two-component conditions indicate that the bis-ureide derivative may be the key intermediate involved in the Biginelli-type reaction using low melting mixtures as solvent and catalyst. Therefore, the combined experimental and theoretical results support that the iminium mechanism is favored in Biginelli reactions.

Acknowledgements. We are grateful to Director, SAIF, Punjab University, Chandigarh for mass spectroscopy. Financial support from UGC, New Delhi for Major Research Project (F 41-281/2012 (SR) is gratefully acknowledged.

\section{REFERENCES}

1. A. Dömling, Chem. Rev., 2006, 106, 17-89.

2. H. G. O. Alvim, E. N. da Silva Júnior and B. A. D. Neto, RSC Adv., 2014, 4, 54282-54299.

3. A. Dömling, W. Wang and K. Wang, Chem. Rev., 2012, 112, 3083-3135.
4. V. G. Santos, M. N. Godoi, T. Regiani, F. H. S. Gama, M. B. Coelho, R. O. M. A. de Souza, M. N. Eberlin and S. J. Garden, Chem. Eur. J., 2014, 20, 12808-12816.

5. C. Graaff, E. Ruijter and R. V. A. Orru, Chem. Soc. Rev., 2012, 41, 3969-4009;

6. B. B. Toure and D. G. Hall, Chem. Rev., 2009, 109, 4439-4486.

7. D. Bonne, T. Constantieux, Y. Coquerel and J. Rodriguez, Chem. Eur. J., 2013, 19, 2218-2231.

8. C. de Graaff, E. Ruijter and R. V. A. Orru, Chem. Soc. Rev., 2012, 41, 3969-4009.

9. S. Brauch, S. S. van Berkel and B. Westermann, Chem. Soc. Rev., 2013, 42, 4948-4962.

10. S. S. Panda, P. Khanna, and L. Khanna, Curr. Org. Chem., 2012, 16, 507-520.

11. A. K. Gupta, N. Singh and K. N. Singh, Curr. Org. Chem., 2013, 17, 474-490.

12. Y. L. Gu, Green Chem., 2012, 14, 2091-2128.

13. P. Slobbe, E. Ruijter and R. V. A. Orru, Med. Chem. Commun., 2012, 3, 1189-1218.

14. M. S. Singh and S. Chowdhury, RSC $A d v$., 2012, 2, 45474592.

15. C. O. Kappe, Eur. J. Med. Chem., 2000, 35, 1043-1052.

16. C. O. Kappe, Tetrahedron, 1993, 49, 6937-6963.

17. P. Lacotte, D. A. Buisson and Y. Ambroise, Eur. J. Med. Chem., 2013, 62, 722-727.

18. A. Crespo, A. El Maatougui, P. Biagini, J. Azuaje, A. Coelho, J. Brea, M. I. Loza, M. I. Cadavid, X. GarcíaMera, H. Gutiérrez-de-Terán and E. Sotelo, ACS Med. Chem. Lett., 2013, 4, 1031-1036.

19. P. Biginelli, Gazz. Chim. Ital., 1891, 21, 337-340.

20. P. Biginelli, Gazz. Chim. Ital., 1893, 23, 360-413.

21. A. S. Paraskar, G. K. Dewkar and A. Sudalai, Tetrahedron Lett., 2003, 44, 3305-3308.

22. K. A. Kumar, M. Kasthuraiah, C. S. Reddy and C. D. Reddy, Tetrahedron Lett., 2001, 42, 7873-7875.

23. M. Gohain, D. Prajapati and J. S. Sandhu, Synlett., 2004, 235-238.

24. C. Simon, T. Constantieux and J. Rodriguez, Eur. J. Org. Chem., 2004, 4957-4980.

25. J. S. Yadav, B. V. S. Reddy, P. Sridhar, S. S. Reddy, K. Nagaiah, N. Lingaiah and P. S. Saiprasad, Eur. J. Org. Chem., 2004, 552-557.

26. H. G. O. Alvim, T. B. Lima, H. C. B. Oliveira, F. C. Gozzo, J. L. Macedo, P. V. Abdelnur, W. A. Silva and B. A. D. Neto, ACS Catal., 2013, 3,1420-1430.

27. P. S. Harikrishnan, S. M. Rajesh, S. Perumal and A. I. Almansour, Tetrahedron Lett., 2013, 54, 1076-1079.

28. H. R. Memarian and M. Soleymani, Ultrason Sonochem., 2011, 18,745-750.

29. G. C. Tron, A. Minassi and G. Appendino, Eur. J. Org. Chem., 2011, 5541-5550.

30. G. Imperato, E. Eibler, J. Niedermaier and B. Konig, Chem. Commun., 2005, 1170-1172.

31. C. Ruß and B. Konig, Green Chem., 2012, 14, 29692982.

32. H. Mahajan, M. Bhardwaj and S. Paul, Org. Prep. Proced. Int., 2014, 46,463-468.

33. G. Kour, M. Gupta, S. Paul,R. Kant and V. K. Gupta, J. Mol. Catal. A: Chem., 2014, 392, 260-269.

34. N. H. Karade, M. Sathe and P. M. Kaushik, Molecules, 2007, 12, 1341-1351. 
